\title{
Real-time lipid patterns to classify viable and necrotic liver tumors
}

Citation for published version (APA):

Vaysse, P. M., Grabsch, H. I., van den Hout, M. F. C. M., Bemelmans, M. H. A., Heeren, R. M. A., Damink, S. W. M. O., \& Siegel, T. P. (2021). Real-time lipid patterns to classify viable and necrotic liver tumors. Laboratory Investigation, 101(3), 381-395. https://doi.org/10.1038/s41374-020-00526-w

Document status and date:

Published: 01/03/2021

DOI:

10.1038/s41374-020-00526-w

Document Version:

Publisher's PDF, also known as Version of record

Document license:

Taverne

Please check the document version of this publication:

- A submitted manuscript is the version of the article upon submission and before peer-review. There can be important differences between the submitted version and the official published version of record.

People interested in the research are advised to contact the author for the final version of the publication, or visit the DOI to the publisher's website.

- The final author version and the galley proof are versions of the publication after peer review.

- The final published version features the final layout of the paper including the volume, issue and page numbers.

Link to publication

\footnotetext{
General rights rights.

- You may freely distribute the URL identifying the publication in the public portal. please follow below link for the End User Agreement:

www.umlib.nl/taverne-license

Take down policy

If you believe that this document breaches copyright please contact us at:

repository@maastrichtuniversity.nl

providing details and we will investigate your claim.
}

Copyright and moral rights for the publications made accessible in the public portal are retained by the authors and/or other copyright owners and it is a condition of accessing publications that users recognise and abide by the legal requirements associated with these

- Users may download and print one copy of any publication from the public portal for the purpose of private study or research.

- You may not further distribute the material or use it for any profit-making activity or commercial gain

If the publication is distributed under the terms of Article $25 \mathrm{fa}$ of the Dutch Copyright Act, indicated by the "Taverne" license above, 


\title{
Real-time lipid patterns to classify viable and necrotic liver tumors
}

\author{
Pierre-Maxence Vaysse ${ }^{1,2,3} \cdot$ Heike I. Grabsch $\mathbb{1}^{4,5}$ - Mari F. C. M. van den Hout ${ }^{4} \cdot$ Marc H. A. Bemelmans ${ }^{2,6}$. \\ Ron M. A. Heeren $\mathbb{1}^{1} \cdot$ Steven W. M. Olde Damink $k^{2,7,8} \cdot$ Tiffany Porta Siegel $\mathbb{B}^{1}$
}

Received: 20 August 2020 / Revised: 30 November 2020 / Accepted: 2 December 2020 / Published online: 22 January 2021

(c) The Author(s), under exclusive licence to United States and Canadian Academy of Pathology 2021

\begin{abstract}
Real-time tissue classifiers based on molecular patterns are emerging tools for fast tumor diagnosis. Here, we used rapid evaporative ionization mass spectrometry (REIMS) and multivariate statistical analysis (principal component analysis-linear discriminant analysis) to classify tissues with subsequent comparison to gold standard histopathology. We explored whether REIMS lipid patterns can identify human liver tumors and improve the rapid characterization of their underlying metabolic features. REIMS-based classification of liver parenchyma (LP), hepatocellular carcinoma (HCC), and metastatic adenocarcinoma (MAC) reached an accuracy of 98.3\%. Lipid patterns of LP were more similar to those of HCC than to those of MAC and allowed clear distinction between primary and metastatic liver tumors. HCC lipid patterns were more heterogeneous than those of MAC, which is consistent with the variation seen in the histopathological phenotype. A common ceramide pattern discriminated necrotic from viable tumor in MAC with 92.9\% accuracy and in other human tumors. Targeted analysis of ceramide and related sphingolipid mass features in necrotic tissues may provide a new classification of tumor cell death based on metabolic shifts. Real-time lipid patterns may have a role in future clinical decision-making in cancer precision medicine.
\end{abstract}

Supplementary information The online version of this article (https:// doi.org/10.1038/s41374-020-00526-w) contains supplementary material, which is available to authorized users.

Tiffany Porta Siegel

t.porta@maastrichtuniversity.nl

1 Maastricht MultiModal Molecular Imaging Institute (M4i), University of Maastricht, Maastricht, The Netherlands

2 Department of Surgery, Maastricht University Medical Center+, Maastricht, The Netherlands

3 Department of Otorhinolaryngology, Head \& Neck Surgery, Maastricht University Medical Center+, Maastricht, The Netherlands

4 Department of Pathology, GROW School for Oncology and Developmental Biology, Maastricht University Medical Center+, Maastricht, The Netherlands

5 Division of Pathology and Data Analytics, Leeds Institute of Medical Research at St. James's, University of Leeds, Leeds, UK

6 GROW School for Oncology and Developmental Biology, Maastricht University Medical Center+, Maastricht, The Netherlands

7 Department of General, Visceral and Transplantation Surgery, RWTH University Hospital Aachen, Aachen, Germany

8 NUTRIM School of Nutrition and Translational Research in Metabolism Faculty of Health, University of Maastricht, Maastricht, The Netherlands

\section{Introduction}

The inherent heterogeneous nature of cancer renders individual patient's response to clinical interventions often unpredictable [1]. Cancer precision medicine aims to improve treatment outcome through precise tumor characterization and tailoring clinical interventions to individual patient's needs [2]. This may be achieved by studying the biological complexity related to tumor heterogeneity at multimolecular levels including genomics [3], proteomics [4], and metabolomics [5]. Patients may benefit from a realtime, cost-effective, and implementable technology for high-throughput tumor characterization. Such a technology could complement the quest for biological understanding and multi-omics integration in precision medicine, [2, 6, 7], which would lead to a more straightforward clinical implementation.

As a subclass of metabolites, lipids are known to be directly involved in defining the tumor phenotype [8]. Their bioactive roles have been studied in detail over the past decades, including in cancer research $[9,10]$. Lipid metabolism reprogramming has been recognized as one of the cancer hallmarks and as a potential therapeutic target [11]. However, cancer precision medicine has not yet considered the full diagnostic potential of lipid metabolism [12, 13]. 
The lipid metabolism underlying metastasis and resistance to cell death may predict disease progression and response to therapy, critical factors for informed clinical decisions.

With the development of real-time intraoperative tools to assist in situ surgical diagnosis, lipid patterns have recently gained interest as tumor-specific metabolic phenotypes [14]. These new tools are able to distinguish tissue constituents based on their physicochemical properties creating tissuespecific lipid patterns for intraoperative tissue classification [15-17]. These real-time classifiers provide intraoperative information about the disease without elaborate processing and expert knowledge, such as required for histopathology, the gold standard for tumor diagnostics. They may, on the one hand, reduce the need for perioperative tissue diagnostics by frozen tissue section analysis and, on the other hand, may provide additional clinically relevant information which cannot be obtained from a hematoxylin- and eosinstained frozen tissue section. Rapid evaporative ionization mass spectrometry (REIMS) analysis is one of the analytical approaches that recently emerged as a tool for intraoperative tissue typing [14-17]. In particular, the combination of surgical handpieces (e.g., diathermia or laser) with REIMS analysis has shown potential to assist surgical tumor resection and in situ tumor diagnostics [18, 19]. REIMS technology can analyze tissue vapors/aerosols and establish their mass spectrometric patterns within a couple of seconds [20]. The thermal disintegration of the tissue produces singly charged molecular ions, a majority of which are lipids. Once ionized, lipids are separated based on their mass-to-charge $(\mathrm{m} / \mathrm{z})$ ratio and their relative abundance is measured. This allows the detection of tissue-specific lipid patterns that can be classified in real time based on preestablished lipid patterns libraries. So far, real-time tissue classifiers have been mainly applied to intraoperative diagnostics. However, lipid patterns generated from tumor tissues may contain information relevant for clinical decisions beyond tissue diagnosis in the operating room.

The lipid patterns may provide insight into the origin of the tumor metastasis. Metastases are the most common cause of cancer death [13] and the liver is one of the main sites of tumor metastases. The invasion-metastasis cascade is a multistep process [21]. Therefore, tumor metastases in the liver are expected to share common molecular alterations, which are different from those of primary liver tumors. In the current study, we compared the lipid patterns of normal liver parenchyma (LP), primary liver tumors (hepatocellular carcinoma (HCC)), and colorectal and breast cancer liver metastases (metastatic adenocarcinoma (MAC)). We hypothesized that the lipid patterns of LP are more similar to that of HCC than that of MAC and that the lipid patterns of MAC are more homogenous than that of HCC.

The implication of bioactive lipids, and especially ceramides, has been extensively reported in tumor cell death mechanism and treatment response [9, 22-24], we therefore included an investigation into the change of lipid patterns between viable and necrotic tumor in our current study hypothesizing that the presence of ceramides can discriminate between viable and necrotic tumor tissue.

\section{Materials and methods}

\section{Patients}

This study included patients who underwent surgery at Maastricht University Medical Center (MUMC + ) between November 2017 and February 2020. Patients older than 18 years were eligible if they were scheduled for partial hepatectomy for removal of a liver tumor or scheduled for removal of a soft tissue tumor. The study was approved by the Medical Ethics Committee of MUMC+ (approval number METC 16-4-168) and conducted according to the revised version of the Declaration of Helsinki. Written informed consent was obtained from each patient prior to study participation.

\section{Tissue procurement}

The resection specimen was transferred fresh from the operating theater to the pathology department as soon as possible. A pathologist dissected normal and tumor tissue surplus to diagnostic needs from the resection specimen for the present study. Tissue slices were used either for immediate REIMS analysis or frozen in liquid nitrogen and stored at $-80^{\circ} \mathrm{C}$ for lipid identification.

\section{REIMS analysis for tissue classification}

After collection, fresh tissue slices were placed on a lowlint, high absorbency and chemically inert wipe (Kimtech, Kimberly Clark), wet with deionized water, and placed on a silicone return electrode (Erbe) (see Supplementary Fig. S1). All experiments were performed in a laminar flow biosafety cabinet (Biowizard Xtra Line, Kojair Blue Series Technologies).

The tissue slides were sampled using a monopolar handpiece (Waters Research Center, Hungary) equipped with a 1-mm-diameter needle electrode connected to a commercially available electrosurgical heat-generator (Force FX, Covidien). Multiple locations within the normal and tumor areas were sampled. Cut and pure modalities were used to cauterize the tissue with a power set between 8 and $25 \mathrm{~W}$ depending on the sample size and the signal quality. The generated electrosurgical vapors were aspirated into a REIMS interface (Waters Corporation, Wilmslow, UK) via a 3-m-long polytetrafluoroethylene tubing, connecting the surgical handpiece and the REIMS interface. 
The REIMS interface was coupled to a mobile Xevo G2-XS quadrupole-time-of-flight mass spectrometer (Q-ToF system, Waters Corporation, Wilmslow, UK).

An aspiration system (ZERO SMOG 2, Weller FT) was set-up to increase the aspiration of the vapors obtained by the venturi tube in the mass spectrometer to avoid the spread of electrosurgical vapors in the laboratory. A MS quality grade isopropanol (Biosolv, Honeywell) solvent was used as a matrix [25] and infused at $150 \mu \mathrm{l} / \mathrm{min}$, containing Leucine-Enkephalin (Leu-Enk, Sigma-Aldrich) in solution for lock-mass correction. The concentration of Leu-Enk ranged from 0.1 to $1 \mathrm{ng} / \mu \mathrm{l}$ and was adjusted on the intensity of the infused [LeuEnk-H] ${ }^{-}$mass peak before tissue analysis. The tissue vapors and the solvent solution were mixed together in a stainless steel capillary toward a heated coil. The electrosurgical vapors were analyzed by REIMS in negative ionization mode and sensitivity mode. Acquisitions were performed over the mass range $\mathrm{m} / \mathrm{z}$ 100-1500. Instrument calibration was performed or checked on sodium formate before tissue analysis. Scan time was set to $1 \mathrm{~s}$. The mass resolution for [LeuEnk-H] was around 50,000.

\section{Histopathological review}

After REIMS analysis, the remaining tissue was fixed in formalin (Unifix, Klinipath) and embedded in paraffin using a standard protocol. Tissue blocks were sectioned at $5 \mu \mathrm{m}$ thickness on a microtome (Leica). Hematoxylin- and eosinstained sections were prepared for histopathology review. During the review, the tissue surrounding the tissue defect from the REIMS procedure was analyzed to predict the type of the evaporated tissue (example in Supplementary Fig. S1C). Representative histology pictures for figures were taken on a microscope by a histopathologist.

\section{REIMS data analysis}

REIMS raw data were imported in the "abstract model builder" software AMX version 0.9.2092.0 (Waters Research Center, Budapest, Hungary). REIMS tissue scans were selected based on their intensities to create a single profile for each sampling spot (one spectrum per sampling spot). For each model, advanced 0.1 binning was selected. Model preprocessing included lock-mass correction, background subtraction, and normalization.

The lipid patterns were subjected to multivariate statistical analysis using a combination of both principal component analysis and linear discriminant analysis (PCA-LDA) as follow:

(1) Unsupervised principal component analysis (PCA) was used for data reduction and to provide an overview of the variance of the lipid patterns included in the model; PCA analysis was performed on a given mass range or on specified mass features of the lipid patterns (as detailed for each analysis).

(2) Supervised linear discriminant analysis (LDA) was used to further optimize the separation between different tissue types by means of projected classes and to reduce the variance within each projected class used for pattern recognition (and therefore for tissue classification).

We compared the tissue classification predicted by PCA-LDA based on the REIMS lipid patterns to the histopathological classification to calculate the accuracy of the lipid patterns based classifier. Cross-validation was performed following a leave-one patient-out classification method. Confusion matrices were generated to assess the accuracy of the tissue classification. PCA score plots provided an overview of the variance of the lipid patterns. Notably, as unsupervised analysis method, the PCA score plots did not consider the histopathological classification, while the LDA added that information to the classifier.

Data matrix from the MAC viable tumor vs. MAC necrosis model was extracted from AMX in Excel and individual mass feature intensities were exported into MetaboAnalyst [26] for statistical analysis as unpaired samples and peak intensity table. No missing values were detected. No data filtering, no sample normalization, no data transformation, and no data scaling were performed. Unequal variance and nonparametric test were selected for univariate analysis by $t$-test ( $p$ value) and box plot to confirm the significant intensity differences between two tissue conditions.

\section{REIMS analysis for lipid identification}

Lipid identification was based on mass accuracy and MS/MS fragmentation. A calibration or a check of the calibration of the instrument REIMS Q-TOF was performed before tissue analysis on the mass range $m / z, 100-1500$ on sodium formate in negative ionization mode. REIMS tissue patterns were corrected on lock-mass deprotonated leucine-encephalin [LeuEnk-H] $]^{-}$at $m / z 554.2615$ and the quality of the calibration was checked on the deprotonated leucine-encephalin dimer [2LeuEnk-H] ${ }^{-}$at $\mathrm{m} / \mathrm{z} 1109.5302$ of the background signal between the tissue samplings. Tandem mass spectrometry experiments were performed on Xevo G2-XS QToF (Waters Corporation, Wilmslow, UK) by collision-induced dissociation with argon gas. Experimental exact masses using external lockmass correction and fragmentation patterns were compared to online database $\mathrm{ALEX}^{123}$ lipid calculator [27] and reference literature [28-30]. In addition, for chlorine adducts isotope 
pattern distributions were checked and compatible with the presence of chlorine in the formula.

\section{Desorption electrospray ionization-mass spectrometry imaging (DESI-MSI) analysis for lipid identification and for lipid in situ distribution}

A tissue block of one tumor sample was taken out of the low temperature freezer, sectioned on a cryotome (Microm) at $8 \mu \mathrm{m}$ thickness. Section was thaw mounted on a regular histology slide (Superfrost, VWR) and stored in a low temperature freezer until further usage. Before DESI-MSI, the tissue section was dried in a desiccator for $20 \mathrm{~min}$. DESI-MSI experiment was performed on a DESI source coupled to a Xevo G2-XS QToF (Waters Corporation, Wilmslow, UK). Solvent was methanol:water (Biosolv) in ratio 98:2. Inlet capillary was heated to approximately 500 degrees Celsius. Acquisition was performed in negative ionization mode, sensitivity mode, over the mass range $\mathrm{m} / \mathrm{z}$ $50-1800$ at $50 \times 50 \mu \mathrm{m}^{2}$ pixel size, with a flow rate of $2 \mu \mathrm{l} /$ min for one scan per second. After experiment, the tissue section was stained by hematoxylin and eosin, scanned with a Mirax Desk digital slide scanner (Zeiss), and annotated by a liver pathologist.

\section{MS analysis for lipid identification}

For identification based on mass accuracy, a calibration of the instrument REIMS Q-TOF was performed before tissue analysis with sodium formate in negative ionization mode. REIMS spectra were corrected on lock-mass deprotonated raffinose $\mathrm{m} / z$. $503.1606[\mathrm{M}-\mathrm{H}]^{-}$. Experimental exact masses were tested on ALEX $^{123}$ lipid calculator [27]. Tandem mass spectrometry experiments were performed on Xevo G2-XS QToF (Waters Corporation, Wilmslow, UK) by collisioninduced dissociation with argon gas. Fragmentation patterns were compared to reference literature [29, 30].

\section{Results}

To study the two hypotheses, we used ex vivo REIMS analysis of electrosurgical vapors to establish the lipid patterns from multiple different locations of fresh resected tissues from 56 patients who had surgery for tumor resection (Supplementary Fig. S1A, B), including 16 primary liver cancer (Supplementary Table S1) and 36 liver metastases (Supplementary Table S2). In total, 2424 lipid patterns were generated with subsequent histopathological tissue review to build a library of tissue-specific lipid patterns (Supplementary Fig. S1C). Details regarding the library of lipid patterns per tissue type and per patient can be found in Supplementary data (Supplementary Fig. S2 and
Supplementary Tables S1-3). Details regarding the clinical and pathological information of the patients included in the study can be found in Supplementary data (Supplementary Tables S4 and 5).

\section{Lipid patterns classify liver parenchyma and liver tumors}

To test the first hypothesis, we studied the classification of LP, primary liver tumor (i.e., HCC), colorectal and breast cancer liver metastases (i.e., MAC). The overall accuracy of the classification of HCC and LP predicted by the PCA-LDA analysis of REIMS lipid patterns compared to the histopathological classification was very good $(96.8 \%)$ but slightly lower than the overall accuracy of classification of MAC and LP (99.4\%) (Supplementary Tables S6 and 7). The classification of LP, HCC, and MAC reached 98.3\% accuracy (Table 1). The classification between HCC and MAC reached 97.4\% accuracy (Supplementary Table S8). These results demonstrate the ability of the lipid pattern library to predict the tissue class with good accuracy. In addition, these results indicate PCA-LDAbased analysis of lipid patterns appears to be better in recognizing MAC than HCC. Representative lipid patterns and pseudo-LDA score plots are shown in Supplementary Fig. S3A-G.

Next, we used the PCA score plots to evaluate the variance of the lipid patterns used for the predicted classification before supervised statistical analysis by LDA. On the PCA score plots with only two tissue classes, HCC and LP lipid patterns were relatively close to each other (Fig. 1A), whereas MAC and LP were relatively well separated (Fig. 1B). Figure 1C supports these observations showing that LP, HCC, and MAC lipid patterns were ordered and aligned along the PC1 axis

Table 1 Confusion matrix for the classification of liver parenchyma (LP), hepatocellular carcinoma (HCC), and metastatic adenocarcinoma (MAC) by rapid evaporative ionization mass spectrometry (REIMS) generated lipid patterns. The tissue classification provided by the histopathologist was used as actual class. Classification of 1743 lipid patterns (1092 LP, $534 \mathrm{HCC}, 117$ MAC) generated from 50 patients using 55 PC dimensions, a mass range $\mathrm{m} / \mathrm{z}, 600-900$ resulted in 30 outliers. The overall accuracy by lipid pattern was $98.3 \%$, ranging from 89.7 to $98.8 \%$ and was highest for LP.

\begin{tabular}{c|c|c|c|c|}
\hline \multirow{2}{*}{$\begin{array}{c}\text { Overall accuracy } \\
\text { 98.3 } \%\end{array}$} & \multicolumn{3}{|c}{ Predicted Class } \\
\hline Actual & LP & HCC & MAC \\
Class & HCC & $4.8 \%$ & $0.3 \%$ & $0.2 \%$ \\
& MAC & $0.9 \%$ & $0.0 \%$ & $89.7 \%$ \\
\hline
\end{tabular}


(PC1 loading plot and suggested identification of some mass features in Supplementary Fig. S3H and Supplementary Table S12). Based on these visual assessments, lipid patterns are more similar between LP and HCC than between LP and MAC.

We included representative histopathological pictures of the three tissue types, used to define the actual class (Fig. 1D-F). While LP and HCC are both mainly composed of hepatocytes and hepatocyte-derived malignant cells,
MAC is composed of malignant epithelial cells originating from the colorectal or the breast primary tumors. The histopathological phenotype of HCC was more similar to that of LP than that of MAC supporting the lipid pattern findings.

In summary, REIMS lipid patterns were able to distinguish between LP, HCC, and MAC and dispersion of the lipid patterns seems to be associated with differences in the histopathological phenotypes.

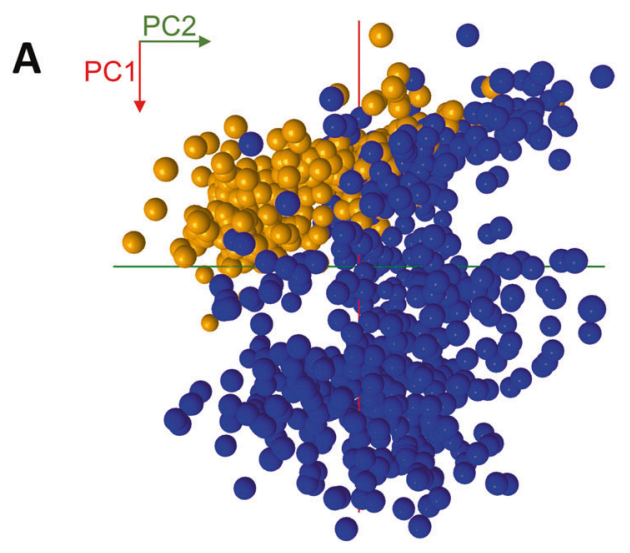

B
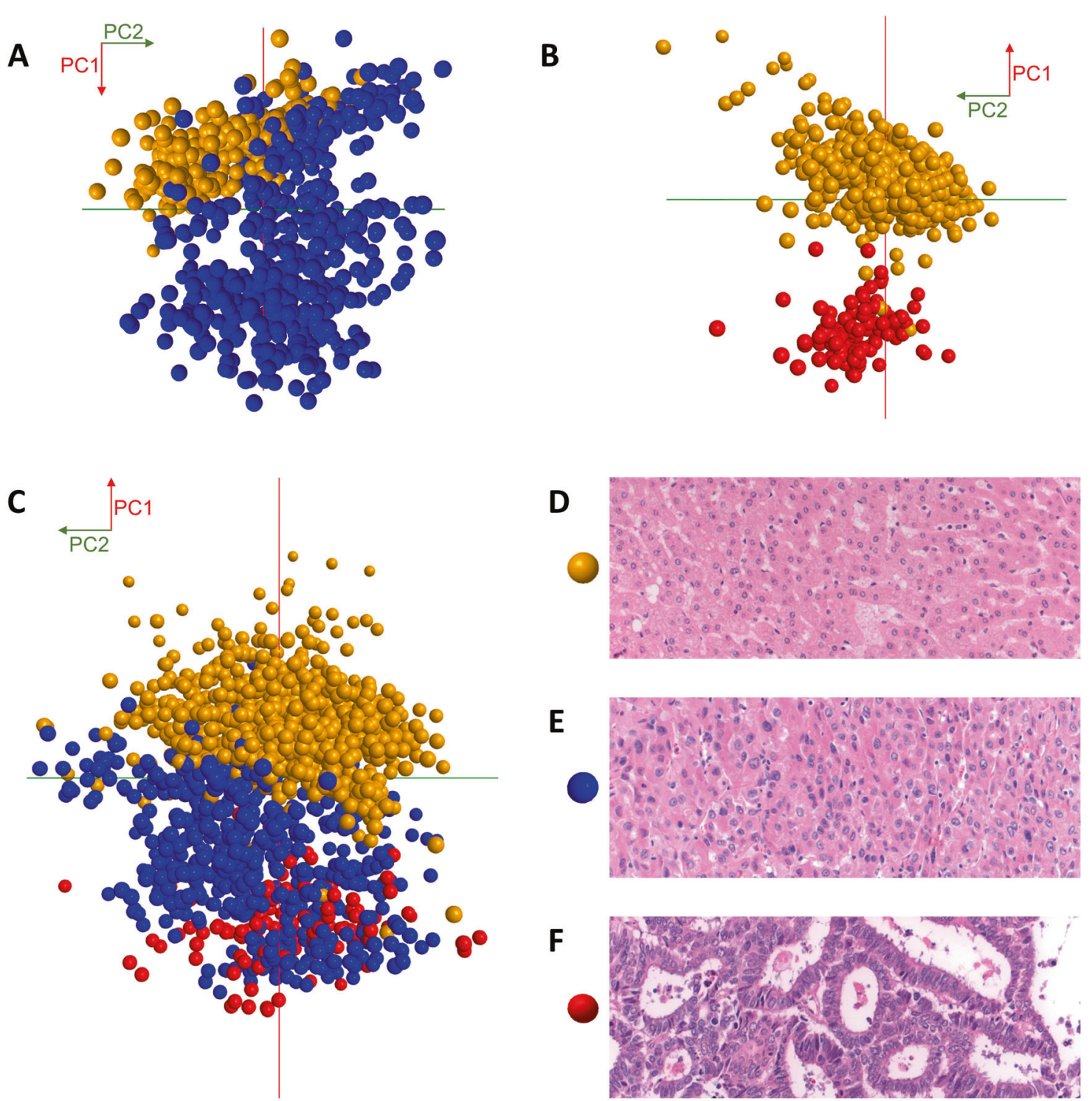

Liver parenchyma (LP) Hepatocellular carcinoma viable tumor (HCC)
Metastatic adenocarcinoma viable tumor (MAC)
Fig. 1 Principal component analysis (PCA) score plots of the lipid patterns of liver parenchyma (LP), hepatocellular carcinoma (HCC), and metastatic adenocarcinoma (MAC) and representative histology. A PCA score plot (PC1 24.4\%, PC2 16.9\%) of 818 patterns (284 LP and $534 \mathrm{HCC}$ ) generated on tissues from 16 patients to differentiate HCC from LP. B PCA score plot (PC1 31.3\%, PC2 16.5\%) of 925 patterns (808 LP and 117 MAC) generated on tissues from 34 patients to differentiate MAC from LP. C PCA score plot (PC1 describes $25.6 \%$ of the total variance of the data, PC2 14.9\%) of 1743 patterns (1092 LP, 534 HCC, 117 MAC) generated on tissues from 50 patients. Representative histology (hematoxylin and eosin (H\&E) staining, original magnification $\times 200$ ) for D LP, $\mathbf{E}$ HCC, and F MAC. 
A

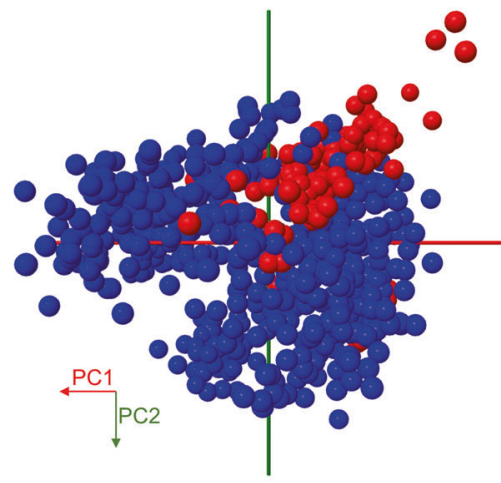

Hepatocellular carcinoma viable tumor (HCC)

Metastatic adenocarcinoma viable tumor (MAC)

C

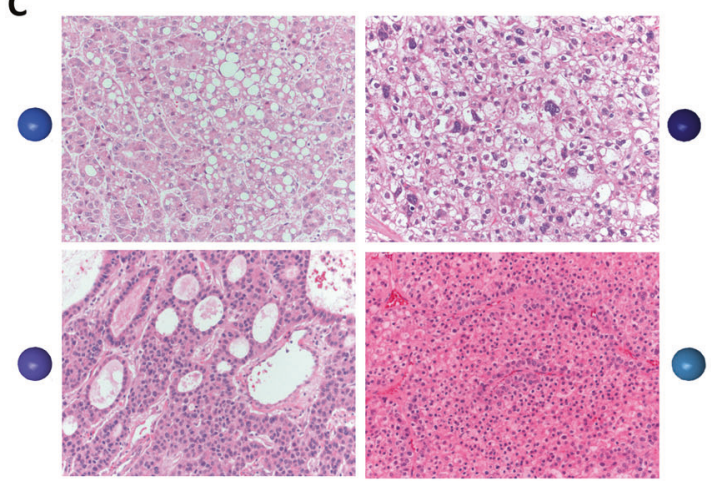

Fig. 2 Tumor type-specific patterns and patient tumor-specific patterns. A Tumor type-specific principal component analysis (PCA) score plot (PC1 23.7\%, PC2 $15.0 \%$, mass range $m / z, 600-900$ ) of 651 lipid patterns (534 hepatocellular carcinoma (HCC), 117 metastatic adenocarcinoma (MAC)) generated on tissues from 35 patients. B Patient-specific PCA score plot (PC1 35.4\%, PC2 $22.3 \%$, mass range $m / z 600-900$ ) of 303 lipid patterns (250 HCC and $53 \mathrm{MAC}$, four

\section{Lipid patterns reflect tumor heterogeneity}

We further compared the variation of the HCC patterns to the MAC patterns as a surrogate of tumor heterogeneity. The visual assessment of the PCA score plot of HCC and MAC showed that the distribution of the HCC lipid patterns is more widespread than the MAC lipid patterns (Fig. 2A) suggesting that lipid patterns within $\mathrm{HCC}$ are more heterogeneous compared to MAC. We selected eight representative tumors to visualize patient-specific tumor lipid patterns (Fig. 2B). The inter-patient variation in lipid patterns was much smaller for MAC compared to HCC (Fig. 2B) suggesting that HCC lipid patterns vary substantially between different patients, whereas the lipid patterns of colorectal cancer metastasis from different patients are very similar. PCA score plots with the lipid patterns for MAC and HCC for all the patients can be found in Supplementary Fig. S4.
B
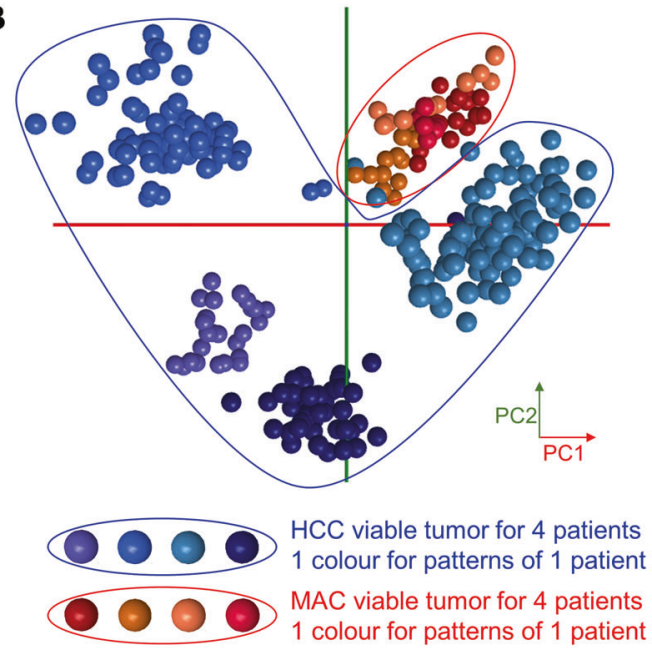

D

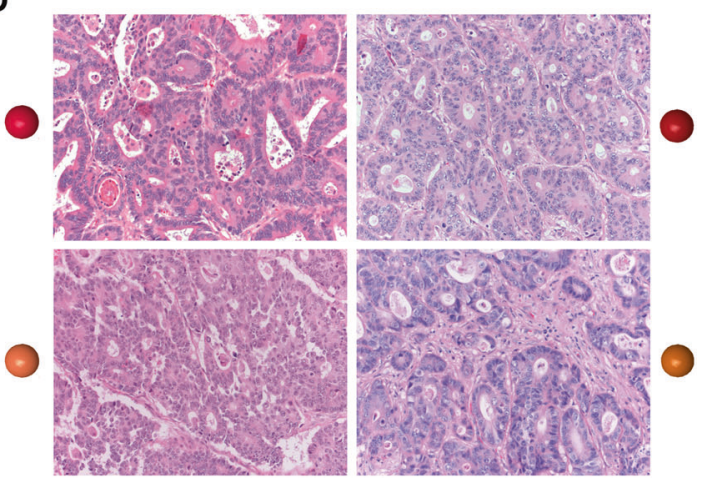

patients per tumor type, eight patients in total). Each color represents the lipid patterns generated from the tissues of one patient. C Representative histopathology for each patient HCC tumor shown in (B) (H\&E staining, original magnification $\times 200)$. D Histopathology for each patient MAC tumor shown in (B) (H\&E staining, original magnification $\times 200$ ).

Furthermore, we compared the histopathological phenotype of the four HCC (Fig. 2C) and the four MAC (Fig. 2D) selected for Fig. 2B. While HCC histology ranged from well differentiated to poorly differentiated including macrotrabecular, glandular-like, and steatohepatitic variants (Fig. 2C), all MAC showed a growth pattern typical for well-differentiated adenocarcinoma (Fig. 2D), suggesting a link between the diversity of lipid patterns and histological phenotypes.

With respect to the heterogeneous lipid patterns observed in the HCCs, we investigated whether the lipid patterns could predict the HCC histological phenotype (steatohepatitic vs. non-steatohepatitic) or potential presence of morphological intra-tumor heterogeneity. Classification of steatohepatic HCC vs. non-steatohepatic HCC was achieved with $91.7 \%$ accuracy (Supplementary Table S9). At least in one of the HCCs, the heterogeneity of the intra-tumor 
morphology appeared to be predictable by lipid patterns as shown in the PCA score plot (Supplementary Fig. S5).

In summary, based on REIMS lipid patterns HCC heterogeneity appears to be more substantial than MAC heterogeneity, with a potential link to histopathological phenotypes. Moreover, REIMS lipid patterns appear to be able to precisely characterize tumors including subtyping.

\section{Discrimination of viable and necrotic tumor parts of metastatic adenocarcinomas}

Primary HCCs but also metastases in the liver can be composed of a mixture of viable and necrotic tumor. Indeed, in colorectal cancer, necrotic debris in the tumor lumen, so-called "dirty necrosis" is characteristic for this tumor type. In other tumors, necrotic tumor is often interpreted as a sign of hypoxia. To test our second hypothesis, we compared 196 lipid patterns obtained from the viable tumor parts to the necrotic parts in MAC of 28 patients (sampling detailed in Supplementary Table S2). The lipid patterns generated via REIMS technology were very different in viable and necrotic MAC and could be distinguished with $92.9 \%$ accuracy (Table 2). Pseudo-LDA score plot, additional PCA and pseudo-LDA score plots, confusion matrices, and test of tissue classifier performed on MAC resected specimens are available in Supplementary data (Supplementary Fig. S6 and Supplementary Tables S10 and 11).

Next, we further investigated the variance of the lipid patterns between viable and necrotic tumor parts. The PCA score plot displayed a separation of MAC and MAC necrosis along the PC1 (explaining $65.0 \%$ of the variance) axis (Fig. 3A). We used the PC1 loading plot to screen the mass features discriminative of MAC necrosis (Fig. 3B). Among these mass features, fatty acids and sphingolipids including ceramides, acylceramides, sphingomyelins, and lactosylceramides were identified (Supplementary Fig. S7 and Supplementary Tables S13 and 14). $t$-tests confirmed a significant increase in MAC necrosis (Supplementary Fig. S8 and Supplementary Table S15). We noticed that these mass features displayed variable intensity ratios in the necrosis patterns between REIMS spectra, including between patients (Fig. 3C, D).

In summary, REIMS lipid patterns are able to distinguish between MAC viable tumor and MAC necrotic tumor. Ceramides and related sphingolipids were among the assigned mass features characteristics for MAC necrotic tumor.

\section{Classification of viable and necrotic tumor from different human tumors}

As ceramides and related sphingolipids discriminated necrotic MAC from viable MAC in diverse intensity ratios, we were interested whether the identified mass features in colorectal metastases are tumor type specific or can also be found in the necrotic part of other tumor types. Therefore, we compared the lipid patterns between viable and necrotic tumor in one HCC, one HCC with neuroendocrine differentiation (HCC NED), one extrahepatic liposarcoma (LPS), and one extrahepatic adrenocortical carcinoma (ACC). Results are shown in Fig. 4. On the PCA score plots, each tumor displayed a separation of viable and necrotic tumor along their PC1 axis. All PC1 loading plots indicated mass features discriminative of necrotic tumor similar to what was observed in necrotic MAC (Fig. 3D). However, the intensity ratios of these mass features differed between tumors (PCA mass features loading plots in Fig. 4).

Noteworthy, we also observed a lipid pattern different from the ceramide pattern to discriminate necrotic from viable tumor in two other HCC and HCC metastasis in the stomach. Details can be found in Supplementary Fig. S9.

In summary, the REIMS lipid pattern distinguishing necrotic MAC from viable MAC appears to be common in multiple human tumors.

Table 2 Confusion matrix for the classification of viable metastatic adenocarcinoma (MAC) and necrotic MAC (MAC necrosis) predicted by lipid patterns. The tissue classification provided by the histopathologist was used as actual class. Classification of 196 patterns (117 viable, 79 necrotic) generated from tissues of 29 patients using 20 principal components dimensions, a mass range $m / z 200-1100$ and resulting in no outlier. The mean class prediction by lipid pattern was $92.9 \%$, ranging from 86.1 to $97.5 \%$ and was best for MAC viable tumor.

\begin{tabular}{|c|c|c|c|}
\hline \multirow{2}{*}{\multicolumn{2}{|c|}{$\begin{array}{c}\text { Overall class rate } \\
\qquad 92.9 \%\end{array}$}} & \multicolumn{2}{|c|}{ Predicted Class } \\
\hline & & MAC & MAC necrosis \\
\hline Actual & MAC & $97.50 \%$ & $2.60 \%$ \\
\hline Class & MAC necrosis & $13.90 \%$ & $86.10 \%$ \\
\hline
\end{tabular}


A

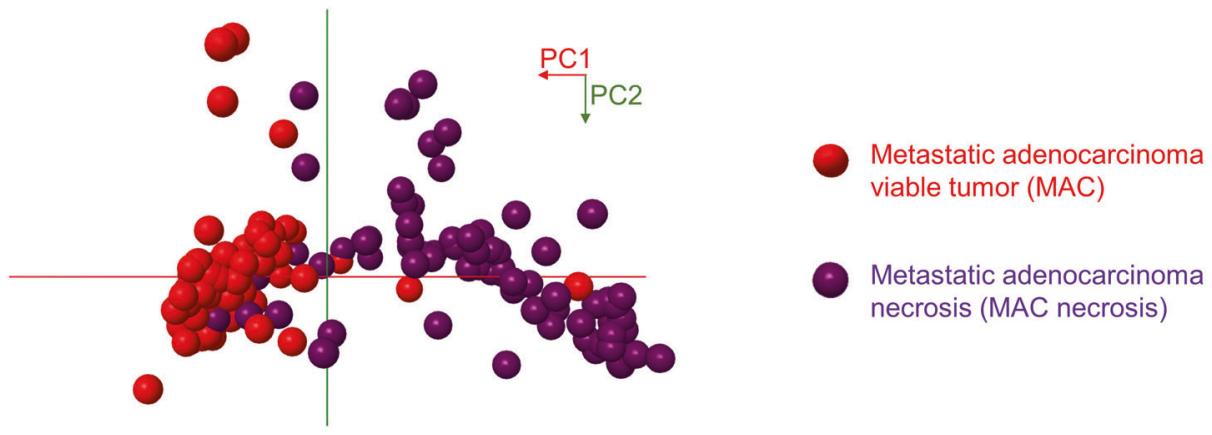

B

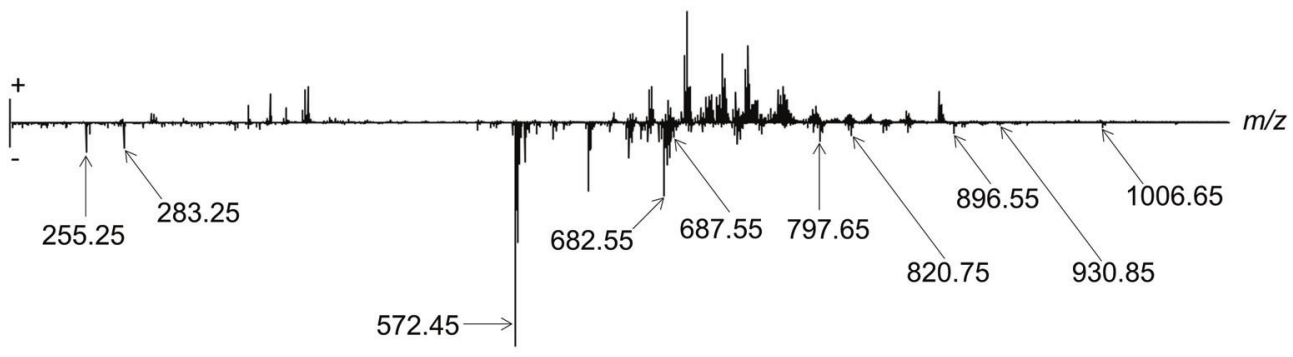

C

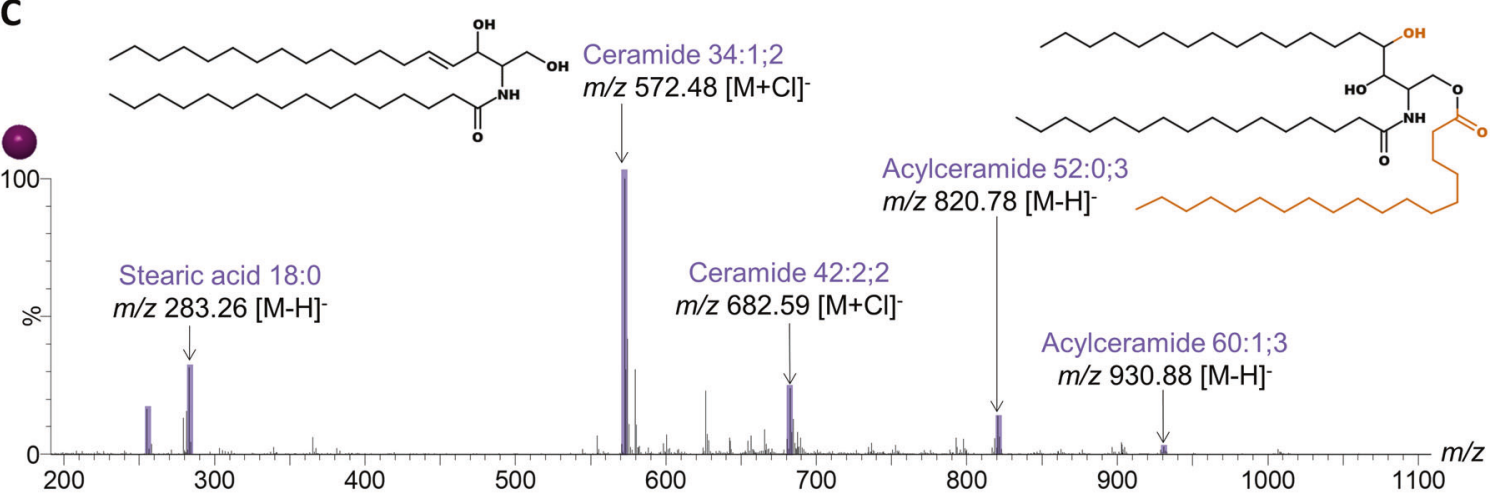

D

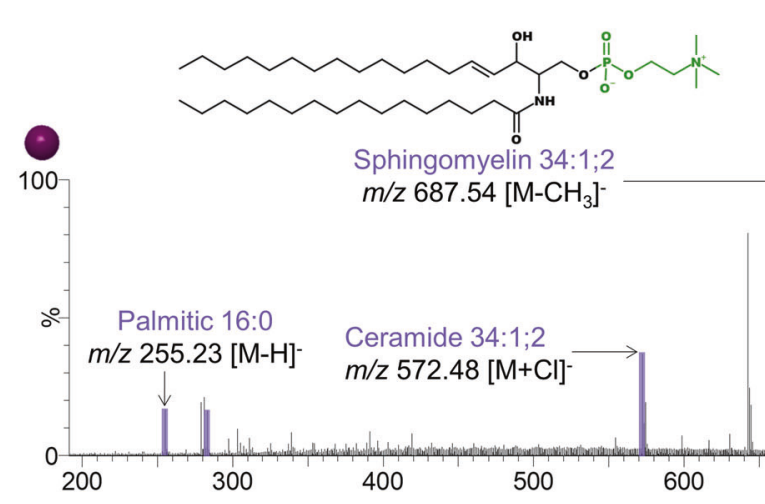

Fig. 3 Discrimination between necrotic and viable metastatic adenocarcinomas. A Principal component analysis (PCA) score plot (PC1 $65.0 \%, \mathrm{PC} 210.3 \%$ ) for the classification of 196 metastatic adenocarcinoma (MAC) lipid patterns (117 MAC (104 colorectal, 11 breast) and $79 \mathrm{MAC}$ necrosis (all colorectal)) generated from tissues of 28 patients using $20 \mathrm{PC}$ dimensions and a mass range $\mathrm{m} / \mathrm{z} 200-1100$. B PC1 loading plot indicating which mass features contributed to the PC1 dimension. C Single rapid evaporative ionization mass spectrometry (REIMS) spectrum for MAC necrosis from one patient.

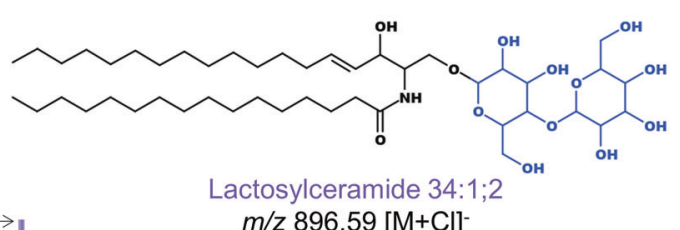
$\mathrm{m} / \mathrm{z} 896.59$ [M+Cl]

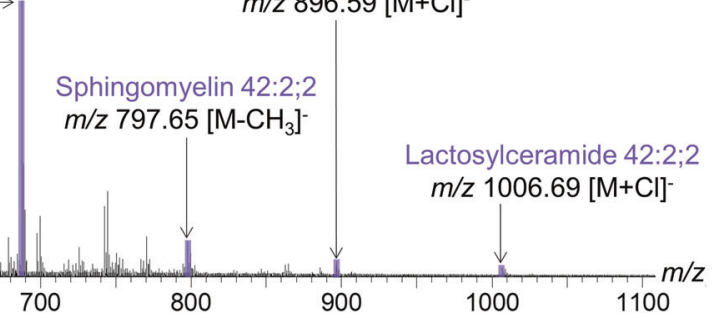

D Single REIMS spectrum for MAC necrosis from another patient. C, D Highlighted in violet mass spectral peaks discriminative of MAC necrosis from MAC based on the PC1 loading plot. Assigned representative molecular structure for mass spectral peaks $\mathrm{m} / \mathrm{z}$ 572.48, $820.78,687.54$, and 896.59 as ceramide $34: 1 ; 2$, acylceramide 52:0;3, sphingomyelin $34: 1 ; 2$, and lactosylceramide $34: 1 ; 2$, respectively. Representative ceramide backbone as $\mathrm{d} 18: 1 / 16 / 0$ in black, diverse moieties in colors. 
A

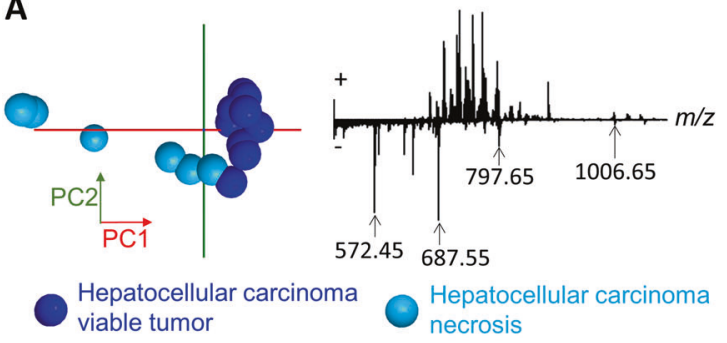

C
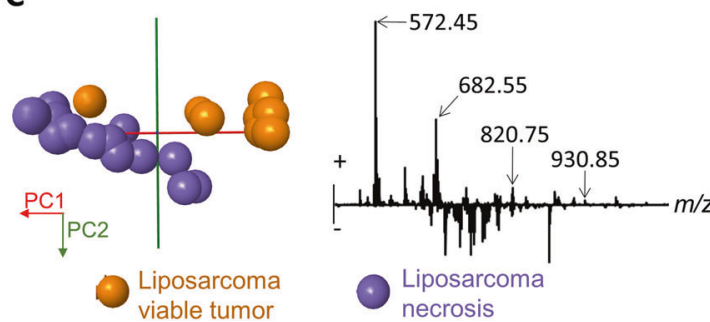

Fig. 4 Discrimination between necrotic and viable tumor. A-D Principal component analysis (PCA) score plots on the left and respective PC1 mass features loading plots on the right (mass range $\mathrm{m} /$ z 500-1100) for: A 19 lipid patterns (13 viable, 6 necrotic) generated from a hepatocellular carcinoma. B Forty-one lipid patterns (18 viable,

\section{Comparisons of necrotic tumors targeted on ceramide metabolic shifts}

As we observed that the same mass features identified as ceramides and related sphingolipids discriminated necrotic from viable tumor in multiple different human tumors, we compared their intensity ratios in necrotic patterns between tumors. We explored the potential of ceramide metabolism from necrotic areas for patient subclassification, independently from the tumor type.

We used PCA score plots of necrotic tumor lipid patterns targeting four specific mass features to evaluate the impact of metabolic shifts involving two ceramides with two acylceramides (Fig. 5A, B), two lactosylceramides (Fig. 5C, D), or two sphingomyelins (Fig. 5E, F). Metabolic links between the mass features used for the PCA were integrated into each PC1/PC2 loading plots (Fig. 5B, D, F) and can be seen in an overview of the sphingolipid pathway in Fig. 6.

We noticed that the orientation of the arrows linking each ceramide and their respective metabolic partner differed. Parallel arrows were displayed only between each ceramide and respective sphingomyelin (Fig. 5F), but this was not the case for acylceramides (Fig. 5B) nor for lactosylceramides (Fig. 5D). Only ceramides and sphingomyelins were linked by a single and direct biochemical reaction.

The necrotic MAC lipid patterns of one of the patients appeared to be more similar to the necrotic tumor lipid patterns of the ACC and the LPS based on the balance ceramides/acylceramides (Fig. 5A). Similar observation was made on the metabolic shift ceramides/lactosylceramides
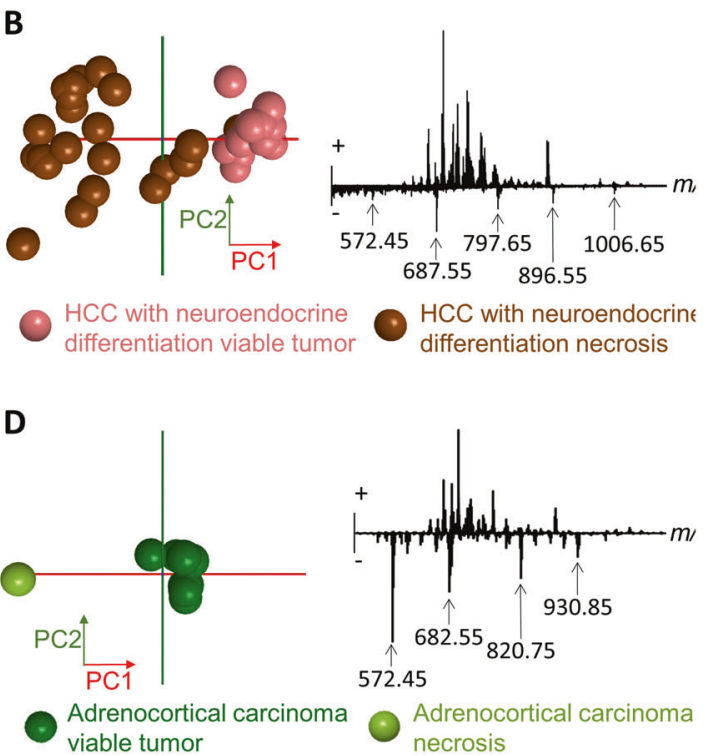

23 necrotic) generated from a hepatocellular carcinoma with neuroendocrine differentiation. $\mathbf{C}$ Twenty-three lipid patterns (9 viable, 14 necrotic) generated from an extrahepatic liposarcoma. D Nine patterns (eight viable, one necrotic) generated from an extrahepatic adrenocortical carcinoma.

for another MAC patient closer to the HCC NED (Fig. 5D). For the ratio ceramides/sphingomyelins, MAC and LPS necrotic patterns were more heterogeneously distributed compared to the necrotic HCC NED patterns (Fig. 5G).

In summary, REIMS lipid patterns offer new perspectives to classify patients based on metabolic shifts present in necrotic tumors.

\section{Discussion}

In current clinical practice, intraoperative decisions often depend on ex vivo examination of resected tissue by a pathologist using frozen tissue sections. Technical issues such as size of the tissue piece that can be investigated during frozen section pathology, type of material (some tissue types cannot be cut frozen), and time constraints during surgery can make frozen sections challenging to interpret with certainty. Besides, the diagnostic delay between excision time and reported diagnosis may hamper the judgment regarding the extension of the resection. Completeness of tumor resection during surgery is usually evaluated by histopathological examination of the fixed resected specimen after surgery, which takes several days. In addition, instant diagnosis of malignancy can be challenging if relevant morphological features are not readily recognizable. Diagnostic subjectivity can lead to disagreement between experts [31]. For definitive diagnosis, histopathology may need to be complemented by immunohistochemistry or other molecular investigations to 

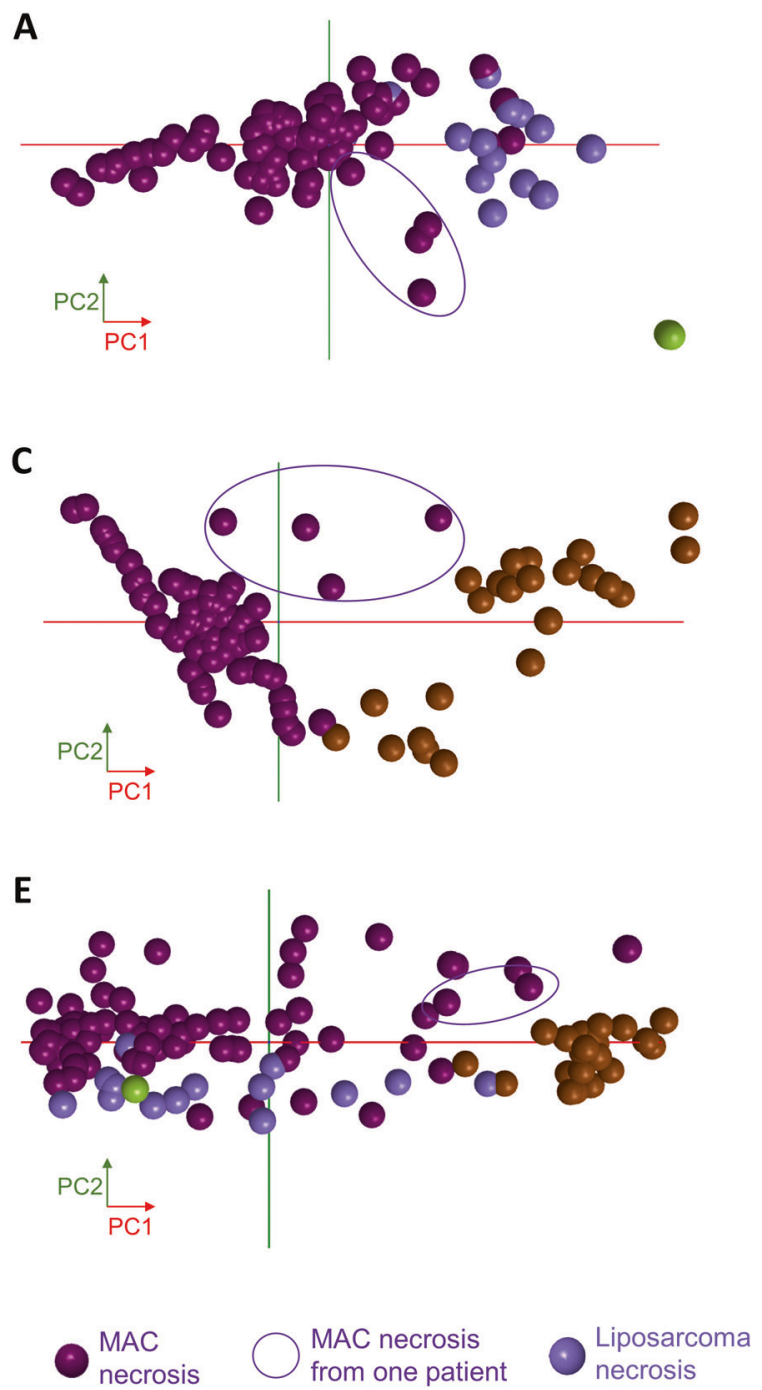

Fig. 5 Comparisons of necrotic tumors based on ceramide metabolic shifts. A, C, E Principal component analysis (PCA) score plots, $\mathbf{B}, \mathbf{D}, \mathbf{F}$ their respective $\mathrm{PC} 1 / \mathrm{PC} 2$ mass features loading plots for lipid patterns of necrotic tumor of: metastatic adenocarcinoma (MAC), liposarcoma (LPS), adrenocortical carcinoma (ACC), hepatocellular carcinoma with neuroendocrine differentiation (HCC NED). A PCA score plot of 94 necrotic patterns (79 MAC, 14 LPS, 1 ACC) targeting four mass features assigned as ceramides $(572.45,682.55)$ and acylceramides $(820.75,930.85)$, generated from tissues of 16 patients $(14$ MAC, 1 LPS, 1 ACC; five PC dimensions, PC1 88.4\%, PC2 11.4\%).

enable the identification of the origin of the tumor extending the specimen reporting time [32].

Near real-time feedback to guide surgical decisions could potentially improve patient care and let to the investigation of fresh tissue classifiers based on lipid patterns. The potential of lipid patterns for the classification of liver tumor tissues has already been suggested using mass spectrometry imaging (MSI) [33] on frozen sections [34, 35], but also REIMS of electrosurgical vapors [17], which allows analysis on fresh resected tissues and a near real-time feedback.
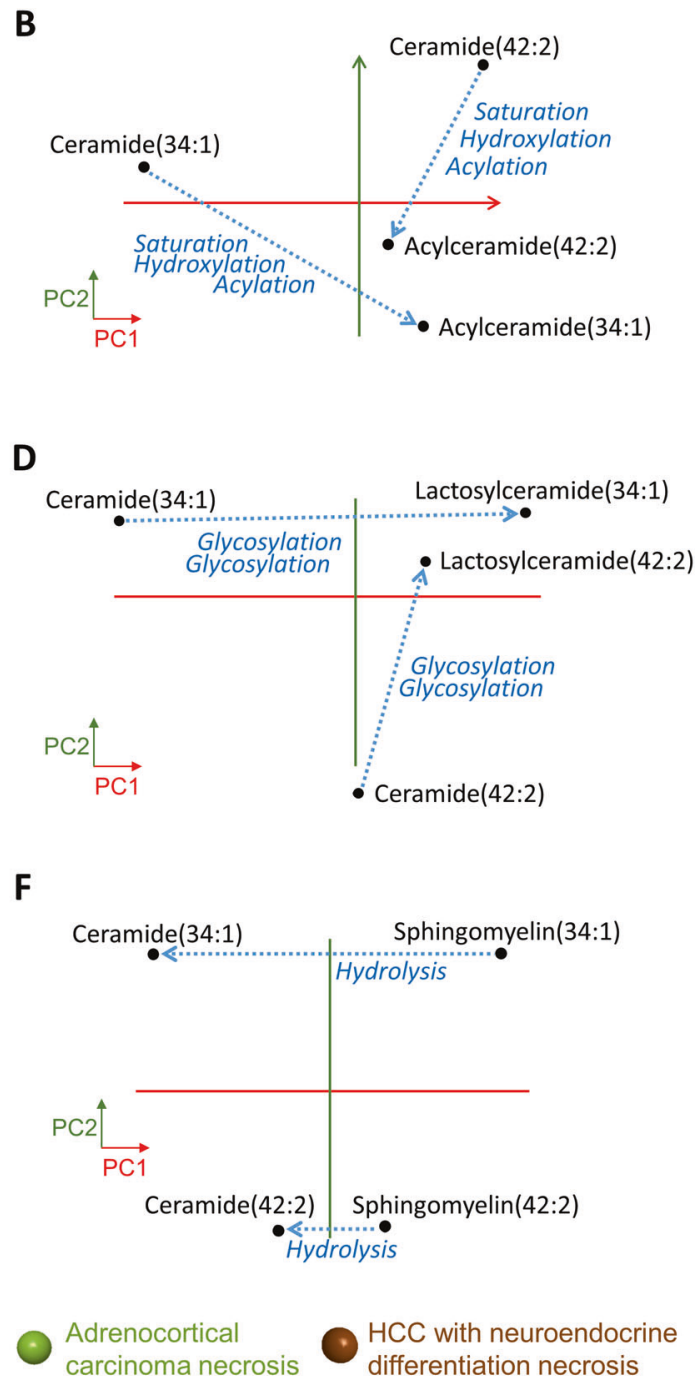

C PCA score plot of 102 necrotic patterns (79 MAC, 23 HCC NED) targeting four mass features assigned as ceramides $(572.45,682.55)$ and lactosylceramides $(896.55,1006.65)$ generated from tissues of 15 patients (14 MAC, 1 HCC NED; five PC dimensions, PC1 86.1\%, PC2 13.7\%). E PCA score plot of 117 necrotic patterns (79 MAC, 1 ACC, 14 LPS, 23 HCC NED) targeting four mass features assigned as ceramides $(572.45,682.55)$ and sphingomyelins $(687.55,797.65)$ generated from tissues of 17 patients (14 MAC, 1 HCC NED, 1 LPS, 1 ACC; five PC dimensions, PC1 95.4\%, PC2 3.7\%).

Our classification of normal LP, HCC, and liver metastasis of colorectal and breast cancer (MAC) based on ex vivo real-time lipid patterns reached $98.1 \%$ accuracy when compared to gold standard pathology suggesting that REIMS technology may be able to assist liver tumor identification during surgery.

Similarly, our investigation demonstrates that lipid patterns provide immediate insights of the tumor metabolic phenotype, which may improve clinical decision. Our experiment was performed ex vivo with a surgical handpiece but dedicated tools using less destructive sampling 


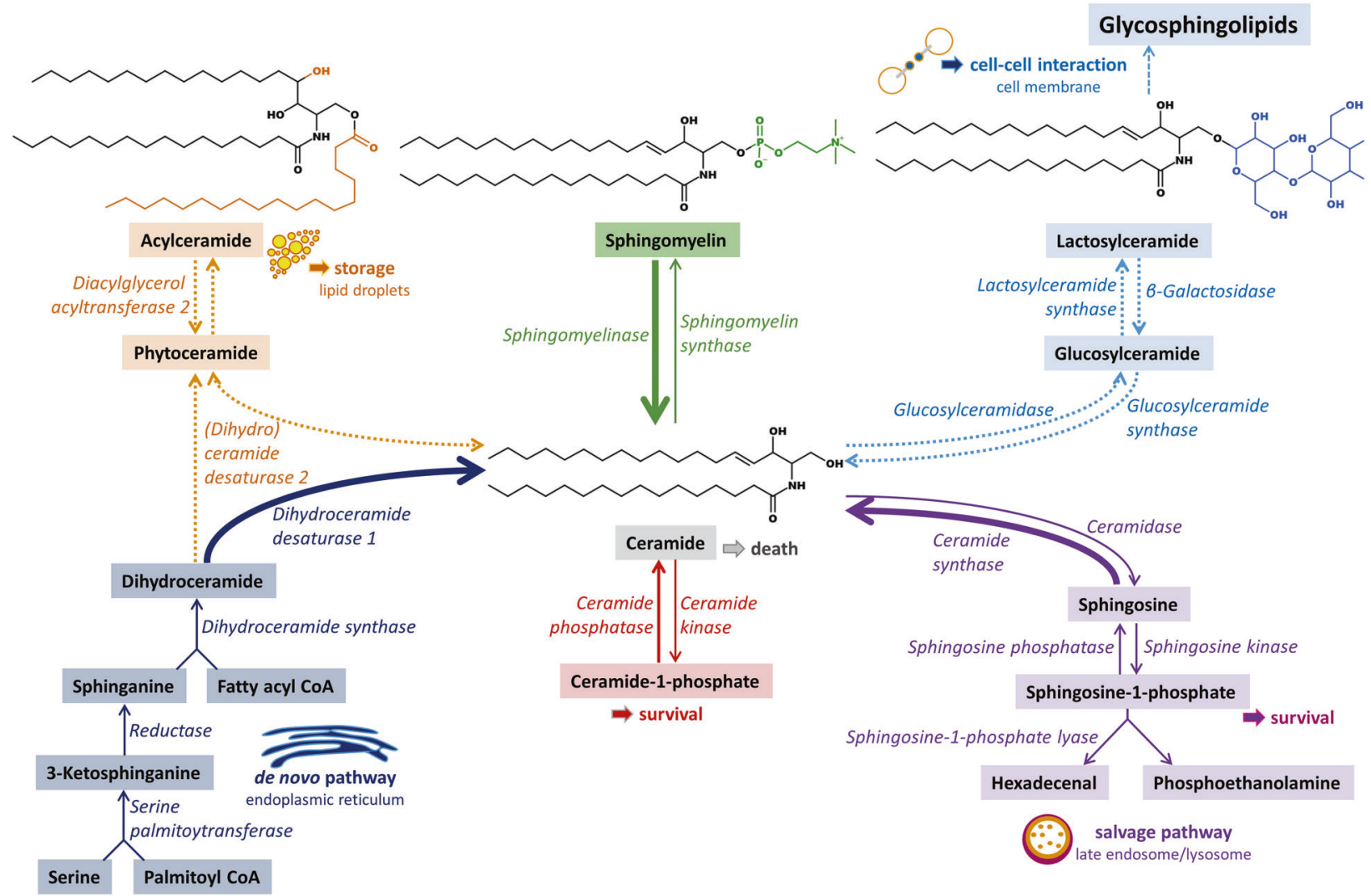

Fig. 6 Scheme of sphingolipid metabolism. Integration of the ceramides and related sphingolipids discriminative of tumor necrosis detected by REIMS with apparent molecular structures (represented

with ceramide backbone d18:1/16/0). Scheme adapted from literature [9, 45-49].

with REIMS or other ionization sources also generating lipid patterns could provide similar conclusions [15, 16, 36]. Likewise, rapid MSI analyses of frozen sections could provide an assessment of the tissue malignancy in a compatible timeframe with intraoperative diagnostics, with the benefit of precise histological correlation [37, 38]. These mass spectrometry technologies based on real-time lipid patterns recognition for cancer precision medicine could be implemented in the operating room or in the pathology laboratory, depending on the impact of the results for clinical decisions. Characterization of tumor heterogeneity could influence clinical interventions (e.g., chemotherapy selection) but could also be conceived as impactful for surgical decisions such as optimized margin resection based on the tumor biology. These perspectives of cancer precision required collaborative efforts for validation of our findings in prospective study on more tissue pieces and investigations about feasibility and implementation. Lipid patterns could translate findings from other research fields such as genomics or proteomics, or correlate directly with detailed clinical data.

Beyond this, the presence of tumor heterogeneity questions the reproducibility of real-time tissue classifiers based on lipid patterns, especially when it comes to compare metabolic phenotypes on a worldwide scale. Beyond hardware and protocol harmonization, cross-validation of tissue classification based on REIMS lipid patterns libraries acquired at multiple sites appears to be one of the next key steps to validate such technology and facilitate its acceptance in clinical practice. Coordinated efforts are key between research and clinical teams to implement the technology within the routine pathology workflow so that input from real-time tissue classifiers can actively support histopathology diagnostics.

Here, we illustrated how lipid patterns can characterize tumor hallmarks on two hypotheses regarding metastasis and necrosis.

For the first hypothesis, we interpreted the overview of the variance of the lipid patterns with regards to tumor heterogeneity and histopathology. While HCC patterns were substantially different between patients (inter-tumor heterogeneity), MAC patterns were more similar between patients (Fig. 2B). The homogenous patterns obtained from MAC compared to the heterogeneous HCC patterns (Fig. 2A) go along with the biological variance expected when comparing primary and secondary tumors. Secondary tumors, as MAC, share common ground biological characteristics to leave their initial generating site, migrate, 
attach, and grow in a new tissue environment [21]. HCC generate more diverse clones of tumors in their initial liver site with multiple features of growth patterns and dedifferentiation with loss of hepatic healthy architecture (Fig. 2C), whereas MAC are generally homogenous and well differentiated in the liver (Fig. 2D).

The generation of patient-specific tumor patterns can be used to evaluate the respective impact of inter- and intratumor heterogeneity on clinical decisions. Further research investigations could contribute to a better understanding of tumor heterogeneity assessment by lipid patterns. The precise spatial distribution of lipids in tissue sections combining MSI [33] and morphometric analysis [39] leads to detail the links between lipid patterns and histopathology changes. We demonstrated that lipid patterns generated by REIMS can provide a fast and quantifiable modality to complement histopathology in the assessment of tumor heterogeneity.

For our second hypothesis, we studied the discrimination of viable and necrotic parts of MAC and other tumors to identify specific lipids involved in tumor cell death metabolism. We used these lipids identified as ceramides and related sphingolipids to classify patient tumors based on specific metabolic shifts, which can be a challenging approach for real-time classifiers.

Real-time classifiers have certain analytical limitations in comparison to the comprehensive characterization of lipid extracts after chromatographic separation [40] for targeted analysis. The detection of lipid species that contribute to the classifier, identification confidence, and their biological interpretation is compromised by the presence of isobaric or isomeric complex lipids, in-source fragmentation, or the lack of resolving power during mass spectrometric analysis. Even if online databases of mass spectra and previous publications generated on diverse platforms are used to confidentially validate lipid identifications, the biological roles of lipids are only partly understood. The still growing knowledge of the role of lipids in tumor biology [9] and the interconnected metabolic pathways each species may experience also represent limitations of biological interpretation. A multitude of diverse and interconnected metabolic pathways are simultaneously active in viable cellular tissues. This makes them more challenging to isolate from each other by mass spectrometry. Before cell death, metabolism might be directed toward essential functional activities, leading to "simplified" necrosis patterns more suitable for investigation. The simultaneous detection of lipids that are metabolically closely related and their statistically significant contribution to discriminate tissues confirm that our findings depict a real-time biological reality.

Moreover, our report of a ceramide pattern discriminative of tumor necrosis by REIMS correlates with previous investigations where ceramides were identified in situ by MSI [33] in necrotic areas of human tumors using diverse analytical platforms. Tata et al. highlighted Cer (34:1) in tissue sections of a variant of a breast cancer cell line xenograft in mice [41]. The authors suggested Cer (34:1) as shared marker for necrosis among various tumors, supported by previous studies on glioblastomas [38]. In addition, Cer(34:1) was reported as part of a lipid pattern in colorectal liver metastasis from 50 patients to distinguish usual necrosis, typical of tumor progression, from infarctedlike necrosis, typical of response to Bevacizumab. Similarly, the distributions of both $\operatorname{Cer}(34: 1)$ and $\operatorname{Cer}(42: 2)$ characterized necrotic areas in high grade serous ovarian cancers [42].

The precise metabolic interconnections of these ceramides and related sphingolipids in tumor cell death are critical to account for and useful to identify targets for cancer therapies. Ceramides are central metabolites of the sphingolipids metabolism (Fig. 6) and their balance with related sphingolipids is critical in the fate of cells for growth and survival [43]. A multitude of cell stress responses to stimuli such as tumor necrosis factor or ultraviolet radiations induce accumulation of ceramides, which lead to a cascade of events that involve the activation of caspases and permeabilization of mitochondrial membranes with release of cytochrome $\mathrm{C}$ to induce cell death [22, 23]. Ceramides can be generated by sphingomyelinase, through de novo or salvage pathways, and their accumulation acts in synergy with chemotherapeutic agents to increase tumor cell death. Pathways that consume the pool of ceramides can be considered pro-survival processes to protect again cell death, enhance resistance to treatment, and contribute to cancer progression [10, 44].

Acylceramides were recently considered a storage form of ceramides in lipid droplets to regulate their accumulation [45]. Increase of acylceramides in colon adenocarcinoma cell lines was associated with more cell survival, and therefore potential resistance to chemotherapy. Structurally, the backbone of the acylceramides in our investigation was assigned to a phytoceramide, limitedly reported in literature [46-48]. An extra hydroxylation and desaturation step in the production of acylceramides from ceramides appears plausible: the extra hydroxyl in the sphingosine backbone was suggested to enable lipid packing in the membrane through an increased amount of hydrogen bounding at the membrane interface [49]. Thus, the storage of acylceramides as phytoceramides seems structurally and biologically rational.

Similarly, the balance between ceramides and lactosylceramides, and glycosphingolipids in general, has been topic of interest in drug therapy, in particular associated to the overexpression of efflux pump P-glycoprotein [50]. Glucosylceramide synthase balances between proapoptotic ceramide and antiapoptotic glucosylceramide are therefore 
the object of studies about drug resistance [24] and drug injury [51].

The delicate balance ceramides/sphingomyelins is indicative of a direct and single biochemical reaction, critical in cell death: the hydrolysis of sphingomyelins into ceramides by sphingomyelinase. In addition to a difference in the phase of the necrosis process, the heterogeneity of this metabolic balance between tumors could be associated with two hypotheses. First, some tumors could require more accumulation of ceramides to die than other tumors. Second, some tumors could generate the ceramides mainly by the sphingomyelinase, while other tumors could mainly use de novo or the salvage pathways.

Moreover, the presence of sphingomyelin as a discriminator of necrosis from viable tumor could suggest that the sphingomyelin breakdown might not be consistently substantial in the generation of ceramides. With regard to patterns changes interpretation, the relative content of the tissue lipid pattern could shift from glycerophospholipids, the main structural lipid class reported by REIMS [17, 18], to sphingolipids during the onset of necrosis. The last catabolic breakdown might lead to the accumulation of remnant end-product lipids that mainly represent the patterns of dead tumor cells. In the absence of functional ceramidases, probably after lysosomes lysis, the ceramide backbone remains intact. Therefore, ceramides and related sphingolipids accumulate in necrotic tissue due to their lipophilic nature that hampers their diffusion [49]. In contrast, the degradation of glycerophospholipids by enzymes such as phospholipases, or spontaneous hydrolyzes, could explain the production of fatty acids that are more prone to diffusion.

Yet, the ceramide pattern is not to be considered a universal pattern for the detection of necrosis in human tumors because we observed a lipid pattern discriminative of necrosis different from the ceramide pattern (Figs. 3 and 4) in two HCC cases (Supplementary Fig. S9). Nevertheless, it appears independent from the tumor origin, whether the tumor originates from ectodermal (i.e., for MAC, HCC) or mesodermal (i.e., LPS, ACC) cells/tissues [52].

Additionally, relative intensity changes of the ceramides in the pattern could provide tumor characteristic molecular information reachable in real time. In our investigation on metabolic shifts, acylceramides were dominant in LPS and ACC necrosis patterns compared to the MAC cohort (Fig. 5A, B). Pharmacokinetics considerations aside, these results appear compatible with the moderate effect of cytotoxic agents to treat these tumors [53, 54]. Likewise, we noticed that the MAC necrosis patterns of one patient presented predominant acylceramides compared to the rest of the MAC cohort. We can hypothesize that the tumor of this patient might react differently to treatment than the rest of the cohort. Similarly, a substantial metabolic shift was noticed between ceramides and lactosylceramides (Fig. 5C, D) suggesting that the full viable tumor might be different metabolically, even if not histologically. As these metabolic shifts do not appear associated with local intra-tumor heterogeneity, this kind of findings could have an impact for clinical decision making.

Our study provides evidence that knowledgeable insights can be obtained from the analysis of necrotic tissues, while the morphologic information to be extracted by a pathologist from a necrotic tissue remains subtler than from viable tissues. The variety of lipid patterns in tumor necrosis could be associated with a different phase or specificity of the metabolic process to reliably provide insight about the metabolism of the viable tumor. In addition, the common detection of necrosis by the same ceramides enables comparisons of tumors from different types based on specific metabolic shifts. It opens new perspective toward translating findings from frequent cancers to rare cancers, far less investigated, to also improve their clinical management.

Lipid patterns generated by REIMS can provide a fast and quantifiable modality to identify and characterize tumor tissue and its heterogeneity. Moreover, we described the ability of real-time analytical technique to reflect cell death, one of the most significant biological processes involved in human diseases. The translational aspect of these findings strengthens the scientific knowledge gathered on lipid metabolism during tumor cell death. The versatility of REIMS allows metabolic analysis of cell models [55] through biological tissues during surgery. In a field governed by tumor heterogeneity affecting patient outcome, precise characterization of heterogeneous tumor metabolic phenotypes is expected to transform clinical decisionmaking.

Our current study demonstrates the benefit of real-time lipid patterns to distinguish between normal and tumor liver tissues which might support intraoperative decision-making in the future. Lipid patterns are informative and potentially clinically useful for prediction of histopathological phenotypes of viable liver tumors, typing of primary and metastatic liver tumors, assessment of tumor heterogeneity, and subtyping of HCCs. Moreover, the report of a ceramide pattern characteristic of tumor necrosis in MAC, and common to multiple human tumors, highlights a potential new approach to use the metabolic phenotype for classification of completely necrotic tumors. This might be particularly interesting to histopathologists and clinicians as the morphological information that can be obtained from necrotic tissues is very limited compared to viable tissues. Cancer precision medicine may benefit from real-time lipid patterns in the future.

Acknowledgements This research was performed at the M4I research institute and was financially supported by Maastricht University 
Medical Center+ and the Dutch Province of Limburg as part of the LINK program. We thank L.J.P.M. Corpelijn (MUMC+) and C.C.C. van Himbeeck (Maastricht University) for their help with patient inclusion and logistics. We thank the teams of the surgery and pathology departments (MUMC+) for their cooperation. We thank J. Balog and S.D. Pringle (Waters) for methodological advice and technical support regarding the utilization of REIMS-qTOF system for clinical studies.

\section{Compliance with ethical standards}

Conflict of interest The authors declare that they have no conflict of interest.

Publisher's note Springer Nature remains neutral with regard to jurisdictional claims in published maps and institutional affiliations.

\section{References}

1. McQuerry JA, Chang JT, Bowtell DDL, Cohen A, Bild AH. Mechanisms and clinical implications of tumor heterogeneity and convergence on recurrent phenotypes. J Mol Med. 2017;95: 1167-78.

2. Pauli C, Hopkins BD, Prandi D, Shaw R, Fedrizzi T, Sboner A, et al. Personalized in vitro and in vivo cancer models to guide precision medicine. Cancer Discov. 2017;7:462-77.

3. Friedman AA, Letai A, Fisher DE, Flaherty KT. Precision medicine for cancer with next-generation functional diagnostics. Nat Rev Cancer. 2015;15:747-56.

4. Zhou L, Wang K, Li Q, Nice EC, Zhang H, Huang C. Clinical proteomics-driven precision medicine for targeted cancer therapy: current overview and future perspectives. Expert Rev Proteomics. 2016;13:367-81.

5. Armitage EG, Ciborowski M. Applications of metabolomics in cancer studies. Adv Exp Med Biol. 2017;965:209-34.

6. Grapov D, Fahrmann J, Wanichthanarak K, Khoomrung S. Rise of deep learning for genomic, proteomic, and metabolomic data integration in precision medicine. Omics. 2018;22:630-6.

7. Olivier M, Asmis R, Hawkins GA, Howard TD, Cox LA. The need for multi-omics biomarker signatures in precision medicine. Int J Mol Sci. 2019;20:4781.

8. Griffin JL, Shockcor JP. Metabolic profiles of cancer cells. Nat Rev Cancer. 2004;4:551-61.

9. Hannun YA, Obeid LM. Sphingolipids and their metabolism in physiology and disease. Nat Rev Mol Cell Biol. 2017;19:175.

10. Ogretmen B. Sphingolipid metabolism in cancer signalling and therapy. Nat Rev Cancer. 2018;18:33-50.

11. Cheng C, Geng F, Cheng X, Guo D. Lipid metabolism reprogramming and its potential targets in cancer. Cancer Commun. 2018;38:27

12. Hanahan D, Weinberg Robert A. Hallmarks of cancer: the next generation. Cell. 2011;144:646-74.

13. Mehlen P, Puisieux A. Metastasis: a question of life or death. Nat Rev Cancer. 2006;6:449-58.

14. Nicholson JK, Holmes E, Kinross JM, Darzi AW, Takats Z, Lindon JC. Metabolic phenotyping in clinical and surgical environments. Nature. 2012;491:384-92.

15. Saudemont P, Quanico J, Robin YM, Baud A, Balog J, Fatou B, et al. Real-TIme Molecular Diagnosis of Tumors Using Waterassisted Laser Desorption/ionization Mass Spectrometry Technology. Cancer Cell. 2018;34:840-51.e4.

16. Zhang J, Rector J, Lin JQ, Young JH, Sans M, Katta N, et al. Nondestructive tissue analysis for ex vivo and in vivo cancer diagnosis using a handheld mass spectrometry system. Sci Transl Med. 2017;9:eaan3968.

17. Balog J, Sasi-Szabó L, Kinross J, Lewis MR, Muirhead LJ, Veselkov K, et al. Intraoperative tissue identification using rapid evaporative ionization mass spectrometry. Sci Transl Med. 2013; 5:194ra193.

18. Phelps DL, Balog J, Gildea LF, Bodai Z, Savage A, El-Bahrawy MA, et al. The surgical intelligent knife distinguishes normal, borderline and malignant gynaecological tissues using rapid evaporative ionisation mass spectrometry (REIMS). $\mathrm{Br} \mathrm{J}$ Cancer. 2018;118:1349-58.

19. Alexander J, Gildea L, Balog J, Speller A, McKenzie J, Muirhead $\mathrm{L}$, et al. A novel methodology for in vivo endoscopic phenotyping of colorectal cancer based on real-time analysis of the mucosal lipidome: a prospective observational study of the iKnife. Surg Endosc. 2017;31:1361-70.

20. Schäfer K-C, Dénes J, Albrecht K, Szaniszló T, Balog J, Skoumal $\mathrm{R}$, et al. In vivo, in situ tissue analysis using rapid evaporative ionization mass spectrometry. Angew Chem. 2009;48:8240-2.

21. Talmadge JE, Fidler IJ. AACR centennial series: the biology of cancer metastasis: historical perspective. Cancer Res. 2010;70: 5649-69.

22. Obeid LM, Linardic CM, Karolak LA, Hannun YA. Programmed cell death induced by ceramide. Science. 1993;259:1769-71.

23. Hernandez-Corbacho MJ, Canals D, Adada MM, Liu M, Senkal $\mathrm{CE}$, Yi JK, et al. Tumor necrosis factor-alpha (TNFalpha)-induced ceramide generation via ceramide synthases regulates loss of focal adhesion kinase (FAK) and programmed cell death. J Biol Chem. 2015;290:25356-73.

24. Kartal Yandim M, Apohan E, Baran Y. Therapeutic potential of targeting ceramide/glucosylceramide pathway in cancer. Cancer Chemother Pharmacol. 2013;71:13-20.

25. Jones EA, Simon D, Karancsi T, Balog J, Pringle SD, Takats Z. Matrix assisted rapid evaporative ionization mass spectrometry. Anal Chem. 2019;91:9784-91.

26. Chong J, Yamamoto M, Xia J. MetaboAnalystR 2.0: from raw spectra to biological insights. Metabolites. 2019;9:57.

27. Pauling JK, Hermansson M, Hartler J, Christiansen K, Gallego $\mathrm{SF}$, Peng B, et al. Proposal for a common nomenclature for fragment ions in mass spectra of lipids. PLoS ONE. 2017;12: e0188394.

28. Murphy RC. Tandem mass spectrometry of lipids: molecular analysis of complex lipids. Royal Society of Chemistry, London, UK; 2015.

29. Murphy RC, Axelsen PH. Mass spectrometric analysis of longchain lipids. Mass Spectrom Rev. 2011;30:579-99.

30. Hsu F-F. Complete structural characterization of ceramides as [M $-\mathrm{H}]-$ ions by multiple-stage linear ion trap mass spectrometry. Biochimie. 2016;130:63-75.

31. Foucar E. Diagnostic decision-making in anatomic pathology. Am J Clin Pathol. 2001;116 Suppl:S21-33.

32. Park JH, Kim JH. Pathologic differential diagnosis of metastatic carcinoma in the liver. Clin Mol Hep. 2019;25:12-20.

33. Vaysse PM, Heeren RMA, Porta T, Balluff B. Mass spectrometry imaging for clinical research-latest developments, applications, and current limitations. Analyst. 2017;142:2690-712.

34. Park YS, Yoo CW, Lee SC, Park SJ, Oh JH, Yoo BC, et al. Lipid profiles for intrahepatic cholangiocarcinoma identified using matrix-assisted laser desorption/ionization mass spectrometry. Clin Chim Acta. 2011;412:1978-82.

35. Nagai K, Uranbileg B, Chen Z, Fujioka A, Yamazaki T, Matsumoto $\mathrm{Y}$, et al. Identification of novel biomarkers of hepatocellular carcinoma by high-definition mass spectrometry: ultrahighperformance liquid chromatography quadrupole time-of-flight mass spectrometry and desorption electrospray ionization mass 
spectrometry imaging. Rapid Commun Mass Spectrom. 2020;34 (Suppl 1):e8551.

36. Genangeli M, Heeren RMA, Porta Siegel T. Tissue classification by rapid evaporative ionization mass spectrometry (REIMS): comparison between a diathermic knife and $\mathrm{CO} 2$ laser sampling on classification performance. Anal Bioanal Chem. 2019;411:7943-55.

37. Basu SS, Regan MS, Randall EC, Abdelmoula WM, Clark AR, Gimenez-Cassina Lopez B, et al. Rapid MALDI mass spectrometry imaging for surgical pathology. NPJ Precis Oncol. 2019;3:17.

38. Calligaris D, Norton I, Feldman DR, Ide JL, Dunn IF, Eberlin LS, et al. Mass spectrometry imaging as a tool for surgical decisionmaking: intraoperative mass spectrometry. J Mass Spectrom. 2013; 48:1178-87.

39. Picard de Muller G, Ait-Belkacem R, Bonnel D, Longuespee R, Stauber J. Automated morphological and morphometric analysis of mass spectrometry imaging data: application to biomarker discovery. J Am Soc Mass Spectrom. 2017;28:2635-45.

40. Luberto C, Haley JD, Del Poeta M. Imaging with mass spectrometry, the next frontier in sphingolipid research? A discussion on where we stand and the possibilities ahead. Chem Phys Lipids. 2019;219:1-14.

41. Tata A, Woolman M, Ventura M, Bernards N, Ganguly M, Gribble A, et al. Rapid detection of necrosis in breast cancer with desorption electrospray ionization mass spectrometry. Sci Rep. 2016;6:35374.

42. Sans M, Gharpure K, Tibshirani R, Zhang J, Liang L, Liu J, et al. Metabolic markers and statistical prediction of serous ovarian cancer aggressiveness by ambient ionization mass spectrometry imaging. Cancer Res. 2017;77:2903-13.

43. Tafesse FG, Holthuis JC. Cell biology: a brake on lipid synthesis. Nature. 2010;463:1028-9.

44. Machala M, Prochazkova J, Hofmanova J, Kralikova L, Slavik J, Tylichova Z, et al. Colon cancer and perturbations of the sphingolipid metabolism. Int J Mol Sci. 2019;20:6051.

45. Senkal CE, Salama MF, Snider AJ, Allopenna JJ, Rana NA, Koller A, et al. Ceramide is metabolized to acylceramide and stored in lipid droplets. Cell Metab. 2017;25:686-97.
46. Rego A, Trindade D, Chaves SR, Manon S, Costa V, Sousa MJ, et al. The yeast model system as a tool towards the understanding of apoptosis regulation by sphingolipids. FEMS Yeast Res. 2014;14:160-78.

47. Mizutani Y, Kihara A, Igarashi Y. Identification of the human sphingolipid C4-hydroxylase, hDES2, and its upregulation during keratinocyte differentiation. FEBS Lett. 2004; 563:93-7.

48. Omae F, Miyazaki M, Enomoto A, Suzuki M, Suzuki Y, Suzuki A. DES2 protein is responsible for phytoceramide biosynthesis in the mouse small intestine. Biochem J. 2004;379:687-95.

49. Gault CR, Obeid LM, Hannun YA. An overview of sphingolipid metabolism: from synthesis to breakdown. Adv Exp Med Biol. 2010;688:1-23.

50. Aouali N, El Btaouri H, Dumontet C, Eddabra L, MalagarieCazenave S, Madoulet C, et al. Accumulation of lactosylceramide and overexpression of a PSC833-resistant P-glycoprotein in multidrug-resistant human sarcoma cells. Oncol Rep. 2011; 25:1161-7.

51. Dupre TV, Doll MA, Shah PP, Sharp CN, Siow D, Megyesi J, et al. Inhibiting glucosylceramide synthase exacerbates cisplatin-induced acute kidney injury. J Lipid Res. 2017; 58:1439-52.

52. Keegan CE, Hammer GD. Recent insights into organogenesis of the adrenal cortex. Trends Endocrinol Metab. 2002;13:200-8.

53. Sperone P, Ferrero A, Daffara F, Priola A, Zaggia B, Volante M, et al. Gemcitabine plus metronomic 5-fluorouracil or capecitabine as a second-/third-line chemotherapy in advanced adrenocortical carcinoma: a multicenter phase II study. Endocr Relat Cancer. 2010;17:445-53.

54. Ducoulombier A, Cousin S, Kotecki N, Penel N. Gemcitabinebased chemotherapy in sarcomas: a systematic review of published trials. Crit Rev Oncol Hematol. 2016;98:73-80.

55. Strittmatter N, Lovrics A, Sessler J, McKenzie JS, Bodai Z, Doria ML, et al. Shotgun lipidomic profiling of the NCI60 cell line panel using rapid evaporative ionization mass spectrometry. Anal Chem. 2016;88:7507-14. 